\title{
Focal points revisited: team reasoning, the principle of insufficient reason and cognitive hierarchy theory
}

Article

Accepted Version

Creative Commons: Attribution-Noncommercial-No Derivative Works 4.0

Bardsley, N. and Ule, A. (2017) Focal points revisited: team reasoning, the principle of insufficient reason and cognitive hierarchy theory. Journal of Economic Behavior and Organization, 133. pp. 74-86. ISSN 0167-2681 doi: https://doi.org/10.1016/j.jebo.2016.10.004 Available at https://centaur.reading.ac.uk/67904/

It is advisable to refer to the publisher's version if you intend to cite from the work. See Guidance on citing.

To link to this article DOI: http://dx.doi.org/10.1016/j.jebo.2016.10.004

Publisher: Elsevier

All outputs in CentAUR are protected by Intellectual Property Rights law, including copyright law. Copyright and IPR is retained by the creators or other copyright holders. Terms and conditions for use of this material are defined in the End User Agreement.

www.reading.ac.uk/centaur 
Central Archive at the University of Reading

Reading's research outputs online 


\title{
Focal Points Revisited: Team Reasoning, the Principle of Insufficient Reason and Cognitive Hierarchy Theory
}

\author{
Nicholas Bardsley† and Aljaž Uleł
}

†Economic and Social Sciences Division, School of Agriculture, Policy and Development, University of Reading, UK; email n.o.bardsley@reading.ac.uk

¥CREED, Amsterdam School of Economics, Universiteit van Amsterdam, The Netherlands; and Faculty of Mathematics, Natural Sciences and Information Technologies, University of Primorska, Slovenia; email a.ule@uva.nl

(C) 2016. This manuscript version is made available under the CC-BY-NC-ND 4.0 license http://creativecommons.org/licenses/by-nc-nd/4.0/ 


\title{
Focal Points Revisited: Team Reasoning, the Principle of Insufficient Reason and Cognitive Hierarchy Theory
}

\author{
Nicholas Bardsley and Aljaž Ule ${ }^{1}$
}

\begin{abstract}
It is well-established that people can coordinate their behaviour on focal points in games with multiple equilibria, but it is not firmly established how. Much coordination game data might be explained by team reasoning, a departure from individualistic choice theory. However, a less exotic explanation is also available based on best-responding to uniform randomisation. We test these two accounts experimentally, using novel games which distinguish their predictions. The results are inconsistent with best-responding to randomisation but consistent with team reasoning as the modal behaviour, though there is also unexplained heterogeneity. Increasing the difficulty of the coordination tasks produces some behaviour suggestive of response to randomisation, but this is a minor feature of the data.
\end{abstract}

\section{Keywords}

Coordination, team reasoning, focal points, bounded rationality

\section{Introduction}

In a classic of modern economics, Schelling (1960) argued persuasively that people have a capacity to coordinate their behaviour on focal points in non-cooperative settings, and that this capacity is mysterious to classical game theory. He based his arguments partly on informal experiments that have since been corroborated in controlled and incentivised settings (Mehta et al. 1994; Bardsley et al. 2010). Several theories have been proposed in response to this evidence. Each departs in some way from classical game theory, but there is no firm consensus yet on how coordination actually occurs. One theory is that play in games with perfectly-aligned payoffs is actually cooperative despite independent decision making, which we term the 'team reasoning' explanation. This explanation departs from received versions of methodological individualism.

\footnotetext{
${ }^{1}$ This research has been supported by grants from Dutch and Slovenian science agencies (NWO grant 451-07031 and ARRS grant J7-6828). We thank two anonymous referees, and seminar participants at the Universities of Amsterdam, Nottingham, Reading and Toulouse for comments which significantly improved the paper.
} 
Since economics is committed to an individualistic account of action, it matters whether or not the team reasoning explanation is needed. Gintis (2003) suggests it is not, because of an alternative explanation: that in situations of unresolved strategic uncertainty people presume and best-respond to random choice. This idea occurs in two accounts of coordination, namely the application of the 'principle of insufficient reason' (Gintis 2003) and a version of 'cognitive hierarchy theory' (CHT) (Bacharach and Stahl, 2000, Camereret al. 2004). ${ }^{2}$ These accounts retain individualism but depart from full rationality. The relevant version of $\mathrm{CHT}$, which we denote $\mathrm{CHT}_{r}$, holds that nonrational choice consists of uniform randomisation. Previous empirical research has not tested the team reasoning explanation against best response to randomisation however; only a version of $\mathrm{CHT}$ based on psychological salience has been tested, which we denote $\mathrm{CHT}_{\mathrm{p}}$. More information on $\mathrm{CHT}_{\mathrm{r}}$ and $\mathrm{CHT}_{\mathrm{p}}$ is given in section 2 .

Classic coordination problems give rise to multiple equilibria, each of which can be rationalized in the standard game-theoretic framework. Both explanations nonetheless yield some determinate predictions there, which have experimental support. We show that, unfortunately, in classic problems their predictions coincide, prohibiting empirical distinction. On the other hand, games that do distinguish them usually introduce payoff asymmetry, and therefore conflicts of interest and social preference considerations. Evidence from these games does not clarify play in the classic problems. Since each cell has identical payoffs for each player, there is neither conflict nor any scope for social preferences to alter the game. We therefore investigate a new experimental game, the 'risky coordination problem', which both distinguishes the two theories and maintains the symmetry of payoffs (Gintis, 2003).

We report two experiments on risky coordination problems. The first implements a simple game which tests the two accounts. The second experiment implements computationally more challenging, but structurally similar games. This is intended to trigger subjects' doubts about the cognitive sophistication of other players, as is depicted in $\mathrm{CHT}$, in order to increase $\mathrm{CHT}_{r}$ 's prospects of success. Results across our two designs are consistent with team reasoning, but not the principle of insufficient reason or $\mathrm{CHT}_{r}$, as a significant driver of coordination. We review the relevant theories and evidence in section 2, and report our experiments in sections 3 and 4. Section 5 provides discussion and interpretation, and section 6 concludes.

\section{Theories of Coordination and Evidence from Coordination Games}

\subsection{Coordination Games}

\footnotetext{
${ }^{2}$ For simplicity we do not observe a distinction in the text between CHT and level -k theory, since they coincide predictively for the games we study.
} 
Informally, a classic coordination problem is one in which two individuals are rewarded for making the same choice from the same alternatives. An example is the heads and tails game, in which two players have to independently nominate the same side of a coin in order to share a prize, and receive nothing otherwise. We restrict our attention to 2-player, one-shot, doubly-symmetric games applying Bardsley et al. (2010)'s formal definition. ${ }^{3}$ The first part of the definition specifies the normal form of the game. Each player chooses a strategy from $S=\left\{s_{1} \ldots S_{n}\right\}$. Payoffs are defined by utility indices $U_{1}, \ldots, U_{n}$ with $U_{(.)}>0$. If each player chooses the same strategy $s_{j}$ then each player receives $U_{j}$, otherwise each receives 0 . If $U_{1}=U_{2}=\ldots U_{n}$ the game is a pure coordination game, if not it is an impure coordination game (or "Hi-Lo" game). To give content to the notion of choosing "the same" strategy where two or more strategies are not distinguishable by outcomes, the second part of the definition stipulates that there is a set $L=\left\{I_{1} \ldots I_{n}\right\}$ of labels which is common knowledge to the players. Players pick their strategy by choosing a label: player $i$ picks strategy denoted $s_{j}$ in the normal form if and only if she chooses $l_{j}$. For simplicity of exposition we refer to the "choice set" to identify L (and thereby S). ${ }^{4}$

In pure coordination games, rewards are the same in each equilibrium. They are described by games with diagonal payoffs equal to 1 and off-diagonal payoffs equal to 0 . Nothing within the payoff structure enables a particular equilibrium to be selected. According to Harsanyi and Selten (1988), in a world of pure rationality, players would therefore coordinate with probability $1 / \mathrm{n}$. Yet in apparent instances of such games, real people do much better (Schelling, 1960; Mehta et al., 1994; Bacharach and Bernasconi, 1997; Bardsley et al. 2010). In Schelling's (1960) informal trials, for example, in the scenario that two people had to meet in New York with no prior arrangement, respondents most commonly nominated Grand Central Station as a meeting place, showing the importance of labels.

Impure coordination games, with variable rewards for coordination and zero payoffs otherwise, seem simpler, perhaps trivial, for real players. But they remain puzzling to analysts, since within the standard framework of common knowledge of rationality the theoretical problem of equilibrium selection still obtains (Regan, 1980; Hollis, 1998; Bacharach 2006). Where there is a payoff dominant equilibrium (PDE), for example, this serves empirically as a strong attractor. Harsanyi and Selten (1988) invoke a "principle" of payoff dominance stipulating that a PDE will be

\footnotetext{
${ }^{3}$ Often a coordination problem is defined more broadly, to include any game with multiple Nash equilibria, including those with asymmetric payoffs (that is, outcomes in which the two players obtain different payoffs). Asymmetry may give rise to conflict of interest and other-regarding concerns that would confound our analysis. Similarly, we do not consider repeated games because they introduce additional factors such as signalling and learning.

${ }^{4}$ In most cases this is unambiguous. However, if a choice set is reported as, for example, \{heads, heads\}, we are reporting the experimenter's explicit labelling. The setting will normally also include contextual features that distinguish each item for the chooser: 'heads on the left' versus 'heads on the right', for example.
} 
selected. But the magnetism of the PDE is unexplained within classical game theory, rendering the principle arbitrary. For it is utility-maximising to choose the PDE strategy if and only if one expects the other to do so with sufficient probability. Since the same also holds for every other strategy, the expectations of PDE strategies are ungrounded. The same problem obtains for other equilibrium refinement concepts, including risk dominance and the maximum of a game's potential function (Monderer and Shapley, 1996). ${ }^{5}$ These concepts coincide with the PDE of impure coordination games, but they do not explain choices.

\subsection{Theories of Coordination}

The literature contains three explanations of coordination: psychological salience, response to randomisation and team reasoning. Taking these in turn, salience is posited by Lewis's (1969) coordination theory as a psychological propensity to choose items that stand out. For example, a shopper faced with dozens of similar toothpastes might choose one with brightly-coloured packaging just because this attracts their attention. Salience acts as a non-rational tie-breaker where actions yield identical expected consequences. This explanation adds a psychological element to game theory. The overall pattern of play is still strategic, however, as players anticipate decisions driven by salience or by anticipation of salience-driven choice.

Psychological salience can be cast as one version of $\mathrm{CHT}$. CHT posits a population structured by different levels of rationality, as formalised by Stahl (1993), Stahl and Wilson (1995) and Camerer et al. (2004). Each player's reasoning belongs to a level, where level 0 players are the least rational and choose non-strategically. Level 1 players optimise based on their beliefs about level Obehaviour. Level 2 players optimise based on their beliefs about the distribution and behaviour of level 0 and level 1 players, and so on. Agents in any level >0 optimise based on beliefs about the rest of the players, who are assumed to belong to lower tiers than themselves. If we assume level 0 players' choices are driven by psychological salience we arrive at $\mathrm{CHT}_{\mathrm{p}}$.

The second explanation involves the idea that an agent might choose randomly, with uniform probability over strategies. In one variant (Gintis 2003), this is posited as the application of the principle of insufficient reason, commonly attributed to Laplace. If strategic players apply this principle in an impure coordination game with strictly ranked equilibria, then each player's best response will be to choose the strategy associated with the PDE. If both players reason in this way, then the agents will coordinate on that outcome. This account amounts to an application of Harsanyi's 'tracing procedure' (Harsanyi and Selten, 1988) with the principle providing the initial beliefs. The players' reasoning is logically dubious (Bjerring, 1978), but can be defended as a version

\footnotetext{
${ }^{5}$ A prediction of risk dominance in $2 \times 2$ games is sometimes explained by invoking hypothetical equi -probable play, as in the $\mathrm{CHT}_{\mathrm{r}}$ argument set out in section 2 .
} 
of bounded rationality. Skyrms (1989), for example, proposes a model in which the players treat consequences of initial information as new information. We are not aware of any empirical assessment of the relevance of principle of insufficient reason in coordination games, however.

The same mechanism occurs under $\mathrm{CHT}_{\mathrm{r}}$, where bounded rationality is explicitly invoked. $\mathrm{CHT}_{\text {r }}$ posits uniform randomisation for level 0 players, and is the most common version of $\mathrm{CHT}$. This has been applied to coordination games by Bacharach and Stahl (2000) in their Variable Frame Level$\mathrm{N}$ Theory (VFLNT). Given unbiased expectations about lower levels' behaviour, $\mathrm{CH}_{\mathrm{r}}$ then makes the same equilibrium prediction as the principle of insufficient reason. For convenience we henceforth refer to both the principle and $\mathrm{CHT}_{r}$ accounts under the term $\mathrm{CHT}_{r}$.

The third explanation is that the individuals, despite making causally independent choices, actually act in concert, asking themselves "What should we do?" According to the team reasoning hypothesis, an individual identifies a profile of strategies which is optimal for her team and then performs her part in it, without conditioning on the other's expected strategy choice. This has been invoked to explain coordination by Bacharach $(1999,2006)$ and Sugden (1995), drawing on Schelling (1960). This explanation departs from a version of methodological individualism usually taken as axiomatic by economists (Elster 1982, 1985), but does not invoke bounded rationality in the sense of cognitive limitations of the agents.

Both team reasoning and $\mathrm{CHT}_{\mathrm{r}}$ have been coupled with a hypothesis about how agents classify their strategies, allowing them to cover a wide variety of cases.

\subsection{Evidence from Coordination Games}

Psychological salience seems consistent with some but not all experimental data. Tests by Mehta et al. (1994) found that choices in pure coordination games with $n>2$ strategies sometimes concentrated on items that were found not to be psychologically salient in independent, parametric choice tasks. For example, when tasked to choose the same integer, subjects tended to choose $\{1\}$, but when asked to nominate any number with no coordination task, $\{1\}$ was not modal. Subsequent experiments by Bardsley et al. (2010), designed to test team reasoning against $\mathrm{CHT}_{\mathrm{p}}$, confirmed this. They also found that subjects' guessing what is psychol ogically salient for others produces different modal choices to the corresponding coordination games. On the other hand, both studies found that for many games $\mathrm{CHT}_{\mathrm{p}}$ apparently performed well. One should also consider that for $2 \times 2$ pure coordination games neither $\mathrm{CHT}_{\mathrm{r}}$ nor team reasoning have any explanatory power, because neither breaks the symmetry of the situation. Thus, psychological salience appears to account for a subset of the data. We confine our attention henceforth to the explanation of the remainder. 
Both $\mathrm{CHT}_{\mathrm{r}}$ and team reasoning can account for data from impure coordination games. For impure coordination games with a PDE, they make the same prediction straightforwardly. For example, in a game with choice set $\{10,9,8\}$ with $U_{1}=10, U_{2}=9$ and $U_{3}=8$, the best strategy combination for the team is $(10,10)$, and therefore team reasoning predicts that each plays $\{10\}$ unconditionally. ${ }^{6}$ Under $\mathrm{CHT}_{r}$, expected payoffs are calculated after assigning probability $1 / 3$ to each of the other's strategies, and the fact that $\{10\}$ offers the highest payoff under this calculation again generates the prediction that $\{10\}$ will be chosen. In this game both accounts thus predict play of the PDE.

For impure coordination games without a unique $\mathrm{PDE}$, both team reasoning and $\mathrm{CHT}_{\mathrm{r}}$ accounts invoke redescriptions of the game. Consider first a game with choice set $\{10,10,10,9\}$, $\mathrm{U}_{1}=\mathrm{U}_{2}=\mathrm{U}_{3}=10$ and $\mathrm{U}_{4}=9$. Empirically, modal choice appears to be $\{9\}$ (Bardsley et al, 2010), so there is no simple regularity that players are attracted by the highest payoffs. Players presumably realize the payoff dominance of coordinating on the same 10, but they risk mis-coordination by picking different 10s, a risk enhanced by the absence of further explicit labelling. Suppose next that the choice set is redefined by each player to be \{any 10,9\}. The on-diagonal expected payoffs of the transformed game are 3.33, and 9. The redefined game therefore has a PDE where both choose $\{9\}$, but this is now predicted by both accounts of coordination.

Next, consider pure coordination games. These can be transformed into impure coordination games if $L$ can be suitably partitioned (requiring $n>2$ strategies). At that point, the same arguments apply. With the choice set \{Ford, Ferrari, Porsche, Jaguar\}, for example, the strategies might be re-categorised as the options \{the ordinary brand, a luxury brand\}. In the recategorised game, selection of \{the ordinary brand\} by each constitutes a dominant equilibrium. Bardsley et al. (2010) report modal choice of the ordinary brand, $\{$ Ford $\}$, in this game, and interpret it as evidence of team reasoning. But this redescription is also posited by VFLNT, which instantiates $\mathrm{CHT}_{r}$, again predicting $\{$ Ford $\}$ for the coordination game.

The availability of two explanations which both invoke players' unobserved re-descriptions of strategies threatens to seriously confound data interpretation in coordination studies. In both Blume and Gneezy (2010) and Crawford et al. (2008) for example, subjects had to coordinate on segments of partitioned discs, one of which is identified as unique by a framing involving shading. In each case, the prediction of coordination on this segment can be derived from either VFLNT or team reasoning. Consequently, essentially the same behaviour is interpreted in Blume and Gneezy's design as evidence of VFLNT, and Crawford et al.'s as evidence of team reasoning. The alternative readings seem equally justified, but invoke completely different modes of reasoning. There is

\footnotetext{
${ }^{6}$ Given symmetric payoffs within cells, it need only be assumed that the team's welfare is a function of both players' payoffs, increasing in both arguments.
} 
therefore a need for games where the two mechanisms of interest yield distinct predictions. We describe and empirically investigate such games in sections 3 and 4 below. 


\section{Experiment 1: Game Play versus Response to Randomisation}

Gintis (2003) describes a variation on a doubly-symmetric coordination game in which team reasoning and $\mathrm{CHT}_{\mathrm{r}}$ make clearly distinct predictions. As in coordination games as defined above, there are multiple equilibria along the leading diagonal of the game matrix, but variable losses are introduced instead of zero payoffs in the off-diagonal cells. We call this a 'risky coordination game' because it introduces risk, in the everyday sense of prospective losses, for coordination failure. With this game we can separate play which is optimised against random behaviour from the PDE. In Gintis's example, each player has to choose an integer in the interval $[1,10]$. If each selects the same integer, each wins that number of monetary units. If different integers are chosen, each loses the larger of the two numbers. This gives rise to the normal form game matrix shown in Figure 1. The game is doubly-symmetric; both players either win or lose the same amount in each cell.

\begin{tabular}{|c|c|c|c|c|c|c|c|c|c|}
\hline 1 & 2 & 3 & 4 & 5 & 6 & 7 & 8 & 9 & 10 \\
\hline 1 & -2 & -3 & -4 & -5 & -6 & $\begin{array}{l}-7 \\
\end{array}$ & -8 & \begin{tabular}{|l|}
-9 \\
\end{tabular} & -10 \\
\hline-2 & 2 & -3 & -4 & -5 & -6 & -7 & -8 & -9 & -10 \\
\hline-3 & -3 & 3 & -4 & -5 & -6 & -7 & -8 & -9 & -10 \\
\hline-4 & -4 & -4 & 4 & -5 & -6 & -7 & -8 & -9 & -10 \\
\hline-5 & -5 & -5 & -5 & 5 & -6 & -7 & -8 & -9 & -10 \\
\hline-6 & -6 & -6 & -6 & -6 & 6 & -7 & -8 & -9 & -10 \\
\hline-7 & -7 & -7 & -7 & -7 & -7 & 7 & -8 & -9 & -10 \\
\hline-8 & -8 & -8 & -8 & -8 & -8 & -8 & 8 & \begin{tabular}{|c|}
-9 \\
\end{tabular} & -10 \\
\hline-9 & -9 & -9 & -9 & -9 & -9 & -9 & -9 & 9 & -10 \\
\hline-10 & -10 & -10 & -10 & -10 & -10 & -10 & -10 & -10 & 10 \\
\hline
\end{tabular}

Figure 1: risky coordination game

Here, choosing larger numbers increases the magnitude of prospective losses given uncertainty about the other's selection. Standard theories of choice under uncertainty, including Expected Utility theory and Prospect Theory (Kahneman and Tversky, 1979), predict that an agent responding to uniform randomisation should choose either $\{2\}$ or $\{3\}$; this prediction carries over to $\mathrm{CHT}_{\mathrm{r}}$ (proof: Appendix 1). The team reasoning prediction is for both to choose $\{10\}$. Gintis (2003) suggests that in this game TR fails comprehensively, but to the best of our knowledgethe evidence for this does not exist. We therefore test the conjecture experimentally. 


\subsection{Experiment 1: Design}

Subjects played the risky coordination game shown in Figure 1. The strategy set for each player consisted of integers in the interval $[1,10]$. In one treatment ('human computer,' or 'HC'), control over the actions of one player in each pair was taken away. Their strategy was determined by computer with uniform probability. The other player in each pair was told that this was how her partner's action would be determined, and had to choose an integer normally. In the second treatment ('human human,' or ' $\mathrm{HH}$ '), the same subjects played under standard game conditions, with each player freely choosing her integer.

Having a computer choose on behalf of a person seems to us better controlled than having subjects play against a computer. For, although the determination of one player's action was shifted to the computer in $\mathrm{HC}$, a social choice situation was maintained, in the sense that each strategy selection affects the payoff of a pair of human subjects.

If coordination proceeds via responses to uniform randomisation, we should observe in $\mathrm{HH}$ the same pattern of choices as in $\mathrm{HC}$, since $\mathrm{HC}$ implements randomness. According to $\mathrm{CHT}_{r}$, any level 0 players will randomise, whilst players in level 1 best-respond to randomisation, in both treatments, choosing from $\{2,3\}$. Higher level players best-respond to randomness in $\mathrm{HC}$ and to mixtures of lower level play in $\mathrm{HH}$, but still choose from $\{2,3\}$ (Appendix 1). If, alternatively, team reasoning is the correct explanation of coordination, we would expect, in contrast, that players choose $\{10\} \mathrm{in} \mathrm{HH}$.

To summarise, in experiment 1 , we test point predictions of each theory plus a comparative prediction of $\mathrm{CHT}_{\mathrm{r}}$ across treatments:

\begin{tabular}{|l|l|l|}
\hline & Theory & Prediction \\
\hline 1 & team reasoning & choice of $\{10\}$ in $\mathrm{HH}$ \\
\hline 2 & $\mathrm{CHT}_{\mathrm{r}}$ & $\begin{array}{l}\text { a. choice of }\{2\} \text { or }\{3\} \text { in both } \mathrm{HH} \text { and } \mathrm{HC} \\
\text { b. identical distributions of choices in } \mathrm{HH} \text { and } \mathrm{HC}\end{array}$ \\
\hline
\end{tabular}

Table 1: predictions for experiment 1

Minor caveats apply to prediction 2. Under $\mathrm{CHT}_{\mathrm{r}}$ (but not the principle of insufficient reason), there should be some level 0 players, who actually randomise uniformly over strategies. Thus, prediction 2a can be stated more precisely for $\mathrm{CHT}_{r}$ as a modal strategy choice of $\{2\}$ or $\{3\}$ with other choices uniformly dispersed. Concerning prediction $2 \mathrm{~b}, \mathrm{CHT}_{\mathrm{r}}$ allows for some switching from $\{2\}$ in $\mathrm{HC}$ to $\{3\}$ in $\mathrm{HH}$ (Appendix 1). 


\subsection{Experiment 1: Procedures}

Experiment 1 was conducted at the CREED laboratory at the University of Amsterdam (UvA) in June 2006, with 44 subjects. Each received a show-up fee of 15 euros, in 30 experimental currency units, from which losses could be deducted. The design was counterbalanced, with half of the subjects playing $\mathrm{HC}$ before $\mathrm{HH}$, and half the opposite order, to control for potential order effects. ${ }^{7}$ Treatment HC was divided into two tasks. In the first task the computer made the choice for one subject in each pair, and in the second task it made the choice for the other subject. Thus, there were three tasks per subject pair, two in $\mathrm{HC}$ and one in $\mathrm{HH}$, and each subject made two choices. The experiment lasted approximately 30 minutes including instructions, comprehension questions and a single sequence of the three tasks. Instructions were repeated to each subject who failed the comprehension check until they were able to correctly answer all questions. No feedback was given on task outcomes or earnings before the end of the experiment. The instructions are given in Appendix 2.

\subsection{Experiment 1: Results}

Figure 2 illustrates the distributions of choices in treatments $\mathrm{HH}$ and $\mathrm{HC}$. We summarize the main observations regarding predictions 1 and 2 in the following results.

Result 1: We find strong support for the team reasoning point prediction.

Most subjects (64\%) chose $\{10\}$ in $\mathrm{HH}$, in line with the team reasoning prediction 1.

Result 2: We find little support for the predictions of $\mathrm{CHT}_{r}$.

The $\mathrm{CHT}_{\mathrm{r}}$ prediction $2 \mathrm{a}$ is rejected since only a small minority of subjects ( $7 \%$ in $\mathrm{HH}$ and $11 \%$ in $\mathrm{HC}$ ) chose $\{2\}$ or $\{3\}$. The modal choice in $\mathrm{HC}$ is $\{1\}$, which is stochastically dominated. If one interpre ts choices of $\{1\}$ in $\mathrm{HH}$ as flawed attempts to best-respond to randomisation, counting $\{1\},\{2\}$ or $\{3\}$ as consistent with $\mathrm{CHT}_{r}$, this would only increase the proportion of subjects complying to $16 \%$. In fact, only one subject chose $\{2\}$ or $\{3\}$ in both conditions, and only 4 subjects chose $\{1\},\{2\}$ or $\{3\}$ in both.

The $\mathrm{CHT}_{\mathrm{r}}$ prediction $2 \mathrm{~b}$ of no difference in choice distributions between treatments is tested with the chi-square test of independence. Since this requires expected cell frequencies of at least 5 (Agresti, 1996), it requires combining response categories into bins. A simple method is to determine the bins from the data as follows. The mode is identified of $\mathrm{HH}$ and $\mathrm{HC}$ choices combined, and bins comprise the mode, integers below it and integers above it. (All data partitions and $\chi^{2}$ tests in this paper, following this approach, are detailed in Table 3, section 4.3.) Here $\{10\}$ is the overall mode

\footnotetext{
${ }^{7}$ No order effects were in fact observed.
} 
and bins comprise $\{10\}$ and $\{[1,9]\}$. We therefore test the null hypothes is of no difference between $\mathrm{HC}$ and $\mathrm{HH}$ using a chi-square test with one degree of freedom. The null hypothesis is rejected $\left(\chi^{2}(1)=23.2 ; p<0.01\right)$. Thus, we find strong evidence against prediction $2 b$. In fact, $68 \%$ of subjects change their choice between $\mathrm{HH}$ and $\mathrm{HC}$.

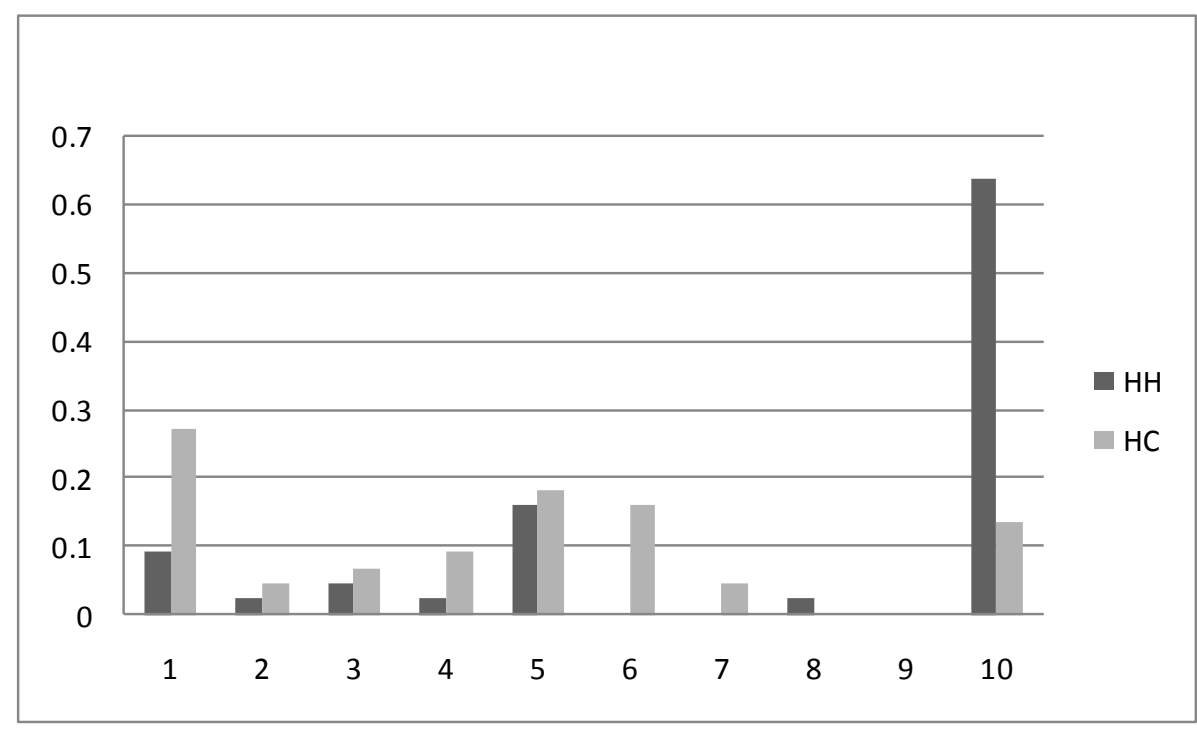

Figure 2: frequency distribution of strategy choices in experiment 1

\subsection{Interpretation of Experiment 1}

The main lesson of experiment 1 is that team reasoning strongly out-performs $\mathrm{CHT}_{r}$ in the game of Figure 1. The very different shapes of the distributions in $\mathrm{HH}$ and $\mathrm{HC}$ make it highly unlikely that $\mathrm{HH}$ choices are based on responses to randomisation. Subjects seem unable to optimise in a one-shot game, since the modal choice in $\mathrm{HC},\{1\}$, is stochastically dominated. As $\{1\}$ is the lowest integer, participants were probably attempting to minimise exposure to loss. However, this description is incomplete, since $\mathrm{HC}$ choices suggest a doubly-censored normal distribution with an interior mode at roughly the mid-point of the strategy space. It is therefore not obvious how best to characterise behaviour in the $\mathrm{HC}$ treatment overall. We also note that around $1 / 3$ of subjects violate the team reasoning point prediction in $\mathrm{HH}$, a substantial amount of unexplained heterogeneity. It could be argued that if these subjects play the same number in both conditions they may be attempting to respond to randomisation, in some flawed manner. Only $18 \%$ of subjects choose the same number (otherthan $\{10\}$ ) however. We also find no association between decisions and the comprehension test performance. 


\section{Experiment 2: Game Play versus Response to Team Reasoning}

Experiment 1 returned evidence favourable to the team reasoning interpretation of coordination, and inconsistent with the $\mathrm{CHT}_{r}$ accounts. A strict falsificationist might conclude that the $\mathrm{CHT}_{r}$ account should be rejected. However, falsificationism has arguably lost ground to views which see empirical work as theory-developing rather than simply theory-refuting (Pawson and Tilley, 1997). The idea of best-responding to random behaviour seems strategically plausible and has empirical support in some experimental contexts ( $\mathrm{Nagel}$, 1995). We therefore conducted a further test on the premise that there are some settings in which best-responding to randomisation will operate and some settings more conducive to team reasoning. The aim of experiment 2 was to gain insight into the conditions under which the $\mathrm{CHT}_{r}$ and team reasoning accounts either succeed or fail, with the goal of informing theory development.

Experiment 2 attempted to undermine team reasoning, and boost consistency with $\mathrm{CHT}_{r}$, by increasing the cognitive difficulty of the coordination problem. The rationale for this is as follows. In a task as computationally easy as the game of experiment 1 , it is perhaps unrealistic to expect there to be a perceived cognitive hierarchy. The 'hierarchy' of $\mathrm{CHT}$, it seems to us, is likely to depend on the cognitive difficulty of the decision problem. Sufficient easiness may lead to perceived cognitive equality, but higher levels of difficulty should give rise to a perceived distribution of abilities. Our auxiliary hypothesis on bounded rationality is that the parameters describing a cognitive hierarchy are endogenous to the choice problem. This is in line with Camerer et al.'s (2004, p863 n1) suggestion that the frequency distribution of player types may be sensitive to the costs and benefits of thinking harder.

Specifically, we propose that in harder tasks the perceived net benefits of deliberation compared to randomisation are diminished, resulting in an increase in the proportion of level 0 type players. Further, actors should be more likely both to anticipate unpredictable behaviour, and responses to unpredictable behaviour, as difficulty increases. We should, then, be more likely to observe responses to randomisation, and less likely to observe team reasoning, in harder tasks. We therefore aimed to induce a cognitive hierarchy by manipulating the difficulty of calculating the team reasoning choice. This was done not to test subjects' maths ability, but to see whether behaviour is more consistent with the $\mathrm{CHT}_{\mathrm{r}}$ account when we depart further from cognitive triviality.

\subsection{Experiment 2: design}

In experiment 1, the comparison of $\mathrm{HH}$ and $\mathrm{HC}$ tests $\mathrm{CHT}_{\mathrm{r}}$. Experiment 2, in contrast, uses the $\mathrm{HC}$ condition to simulate team reasoners, so that the treatment comparison tests team reasoning. One reason for this was to detect strategic switching to low numbers in $\mathrm{HH}$ (prediction $4 \mathrm{~b}$ below). Also, 
we wanted to evaluate the two theories in a similar manner, by letting each one represent the null hypothesis in an experimental test. In treatment $\mathrm{HC}$, then, the integer of one of the paired players was predetermined according to the team reasoning prediction. The other player was told that the computer would select the number which gives the highest joint earnings if both participants choose it. In HC, therefore, the choosing subject has to respond to team reasoning. If team reasoning is the only non-random process at work in $\mathrm{HH}$, choices in $\mathrm{HH}$ and $\mathrm{HC}$ should be realisations of the same underlying distribution. According to $\mathrm{CHT}_{r}$, in contrast, players in levels $>0$ can solve the team reasoning computational problem but still best-respond to randomisation in the interpersonal game setting. So $\mathrm{CHT}_{r}$ predicts choices of lower integers in $\mathrm{HH}$ than in $\mathrm{HC}$.

Three doubly-symmetricgames were used. They shared the feature with Experiment 1, that if the paired subjects chose different integers, they would both lose the larger number in currency units. If their chosen integers matched they would earn positive amounts. The winning amounts may, however, differ from the face value of the chosen integers, as set out below:

a) 'Low' difficulty. Matches on prime numbers pay their face value, while matches on other integers pay half their face value.

b) 'Medium' difficulty. A match on $x$ pays its face value, where $x=8 ! / 7 !$, while matches on all other integers pay half their face value.

c) 'High' difficulty. A match on $x$ pays its face value, where $\sqrt[x]{59049}=9$, while matches on all other integers pay 4.

As the labelling indicates, the tasks were constructed to increase difficulty of team reasoning from a) to c). Subject recruitment was not restricted to courses with mathematical content. We therefore expected that there would be considerable variation in participants' problem solving ability, and, therefore, good prospects of observing responses to randomisation in $\mathrm{HH}_{\text {. }} \mathrm{CHT}_{\mathrm{r}}$ predicts low number choices for $\mathrm{HH}$, with the exact prediction varying slightly between games as specified below. These tasks give rise to the normal form game matrices shown in Figure 3 below.

An additional motivation for experiment 2 was to eliminate the possibility that subjects in $\mathrm{HH}$ are coordinating on salient features of the strategy space in something other than the team reasoning sense. For example, in experiment 1 , it is conceivable that 10 is simply a psychologically salient number. To exclude this possibility, the strategy space is kept the same in each variant. Number salience is therefore held constant, whist team reasoning selects a different integer in each case. 


\begin{tabular}{|c|c|c|c|c|c|c|c|c|c|}
\hline & & & & Low & & & & & \\
\hline 1 & 2 & 3 & 4 & 5 & 6 & 7 & 8 & 9 & 10 \\
\hline 1 & -2 & -3 & -4 & -5 & -6 & -7 & -8 & -9 & -10 \\
\hline-2 & 2 & -3 & -4 & -5 & -6 & -7 & -8 & -9 & -10 \\
\hline-3 & -3 & 3 & -4 & -5 & -6 & -7 & -8 & -9 & -10 \\
\hline-4 & -4 & -4 & 2 & -5 & -6 & -7 & -8 & -9 & -10 \\
\hline-5 & -5 & -5 & -5 & 5 & -6 & -7 & -8 & -9 & -10 \\
\hline-6 & -6 & -6 & -6 & -6 & 3 & -7 & -8 & -9 & -10 \\
\hline-7 & -7 & -7 & -7 & -7 & -7 & 7 & -8 & -9 & -10 \\
\hline-8 & -8 & -8 & -8 & -8 & -8 & -8 & 4 & -9 & -10 \\
\hline-9 & -9 & -9 & -9 & -9 & -9 & -9 & -9 & 4.5 & -10 \\
\hline-10 & -10 & -10 & -10 & -10 & -10 & -10 & -10 & -10 & 5 \\
\hline
\end{tabular}

Medium

\begin{tabular}{|c|c|c|c|c|c|c|c|c|c|}
\hline 1 & 2 & 3 & 4 & 5 & 6 & 7 & 8 & 9 & 10 \\
\hline 0.5 & -2 & -3 & -4 & -5 & -6 & -7 & -8 & -9 & -10 \\
\hline-2 & 1 & -3 & -4 & -5 & -6 & -7 & -8 & -9 & -10 \\
\hline-3 & -3 & 1.5 & -4 & -5 & -6 & -7 & -8 & -9 & -10 \\
\hline-4 & -4 & -4 & 2 & -5 & -6 & -7 & -8 & -9 & -10 \\
\hline-5 & -5 & -5 & -5 & 2.5 & -6 & -7 & -8 & -9 & -10 \\
\hline-6 & -6 & -6 & -6 & -6 & 3 & -7 & -8 & -9 & -10 \\
\hline-7 & -7 & -7 & -7 & -7 & -7 & 3.5 & -8 & -9 & -10 \\
\hline-8 & -8 & -8 & -8 & -8 & -8 & -8 & 8 & -9 & -10 \\
\hline \begin{tabular}{|c|}
-9 \\
\end{tabular} & \begin{tabular}{|c|}
-9 \\
\end{tabular} & -9 & \begin{tabular}{|c|}
-9 \\
\end{tabular} & -9 & \begin{tabular}{|c|}
-9 \\
\end{tabular} & -9 & -9 & 4.5 & -10 \\
\hline-10 & -10 & -10 & -10 & -10 & -10 & -10 & -10 & -10 & 5 \\
\hline
\end{tabular}


High

\begin{tabular}{|c|c|c|c|c|c|c|c|c|c|}
\hline 1 & 2 & 3 & 4 & 5 & 6 & 7 & 8 & 9 & 10 \\
\hline 4 & -2 & -3 & -4 & -5 & -6 & -7 & -8 & -9 & -10 \\
\hline-2 & 4 & -3 & -4 & -5 & -6 & -7 & -8 & -9 & -10 \\
\hline-3 & -3 & 4 & -4 & -5 & -6 & -7 & -8 & -9 & -10 \\
\hline-4 & -4 & -4 & 4 & -5 & -6 & -7 & -8 & -9 & -10 \\
\hline-5 & -5 & -5 & -5 & 5 & -6 & -7 & -8 & -9 & -10 \\
\hline-6 & -6 & -6 & -6 & -6 & 4 & -7 & -8 & \begin{tabular}{|c|}
-9 \\
\end{tabular} & -10 \\
\hline-7 & -7 & -7 & -7 & -7 & -7 & 4 & -8 & -9 & -10 \\
\hline-8 & -8 & -8 & -8 & -8 & -8 & -8 & 4 & -9 & -10 \\
\hline-9 & -9 & -9 & -9 & -9 & -9 & -9 & -9 & 4 & -10 \\
\hline-10 & -10 & -10 & -10 & -10 & -10 & -10 & -10 & -10 & 4 \\
\hline
\end{tabular}

Figure 3: risky coordination games in experiment 2

To summarise, in experiment 2, we test point predictions and treatment predictions for both theories, plus the hypothesis on bounded rationality:

\begin{tabular}{|l|l|l|}
\hline & Theory & Prediction \\
\hline 3 & team reasoning & $\begin{array}{l}\text { a. choice of }\{7\} \text { in Low, }\{8\} \text { in Medium and }\{5\} \text { in High in } \mathrm{HH} \\
\text { b. identical distributions of choices in } \mathrm{HC} \text { and } \mathrm{HH}\end{array}$ \\
\hline 4 & $\mathrm{CHT}_{\mathrm{r}}$ & $\begin{array}{l}\text { a. choice of }\{2\} \text { or }\{3\} \text { in Low , }\{2\} \text { in Medium, and }\{1\} \text { or }\{2) \\
\text { in High, in } \mathrm{HH} \\
\text { b. lower choices in HH compared to HC }\end{array}$ \\
\hline 5 & $\begin{array}{l}\text { hypothesis on } \\
\text { bounded } \\
\text { rationality }\end{array}$ & $\begin{array}{l}\text { a. relative performance of prediction 3a over 4a } \\
\text { deteriorates across Low, Medium and High conditions } \\
\text { b. relative performance of prediction 3b over 4b } \\
\text { deteriorates across Low, Medium and High conditions }\end{array}$ \\
\hline
\end{tabular}

Table 2: predictions for experiment 2

\subsection{Experiment 2: Procedures}

Experiment 2 was conducted at the CREED laboratory at the University of Amsterdam, in June 2010 and June 2011. Each subject was given a show-up fee of 15 euros, in 30 experimental currency units. Separate samples were drawn from the same student population for Low, Medium and High. Sample sizes were 30, 28 and 32 respectively. All subjects played treatment $\mathrm{HH}$ first and $\mathrm{HC}$ second in order 
to avoid biasing $\mathrm{HH}$ decisions in favour of team reasoning. Instructions for each part of the experiment were given only after the previous part was finished. As in experiment 1 , treatment $\mathrm{HC}$ was divided in two tasks and each subject played HC once actively, and once passively with the computer making her decision. The computer chose according to team reasoning. Thus, there were three tasks per subject, two of which involved decision making. The experiment lasted approximately 30 minutes including instructions, comprehension questions and one sequence of the three tasks. No feedback was given on outcomes or earnings before the end of the experiment. Instructions are given in Appendix 2.

\subsection{Experiment 2: Results}

Figure 4 illustrates the distributions of choices in treatments $\mathrm{HH}$ and $\mathrm{HC}$. We summarize the main observations regarding predictions $3-5$ in the following results.

Result 3. Point predictions of team reasoning are supported but its treatment prediction fails.

The team reasoning point prediction $3 \mathrm{a}$ is strongly modal for choices in $\mathrm{HH}$ in each game, with $46 \%$, $50 \%$ and $50 \%$ of subjects making this choice in Low, Medium and High respectively. Its prediction $3 \mathrm{~b}$ is tested with a chi-squared test (Table 3). This is not significant at the $5 \%$ level for any of the three tasks, but is significant at the $10 \%$ level for Medium and $\operatorname{High}\left(\chi^{2}(2)=1.1, p=0.57 ; \chi^{2}(2)=5.9, p=\right.$ $0.05, \chi^{2}(2)=5.4, p=0.07$ respectively). However, combining data from the three games results in a strong rejection of the null hypothesis $\left(\chi^{2}(2)=9.5 ; p<0.01\right)$. Thus, prediction $3 b$ fails.

Result 4. Point predicitons of $\mathrm{CHT}_{r}$ are rejected but its treatment prediction is supported.

Prediction 4a fares poorly in comparison to 3a, with relatively few subjects in $\mathrm{HH}$ choosing according to the $\mathrm{CHT}_{\mathrm{r}}$ point prediction. $7 \%$ of subjects conform to this prediction in Low, $4 \%$ in Medium and $25 \%$ in High. However, as in experiment 1 , one might interpret choices of $\{1\}$ in Low and Medium as flawed attempts at $\mathrm{CHT}_{r}$. This would alter the proportions in Low and Medium to $13 \%$ and $29 \%$ respectively.

For $\mathrm{CHT}_{\mathrm{r}}$ 's treatment prediction $4 \mathrm{~b}$, a binomial test across the three games assesses whether subjects who change their choice between $\mathrm{HC}$ and $\mathrm{HH}$ do so randomly. 35 subjects changed their decisions, with 26 of these choosing a lower number in $\mathrm{HH}$. The null hypothesis that switches to higher and lower numbers are equi-probable is rejected (2-tailed binomial test, $p<0.01$ ). Thus, $4 \mathrm{~b}$ is supported. 

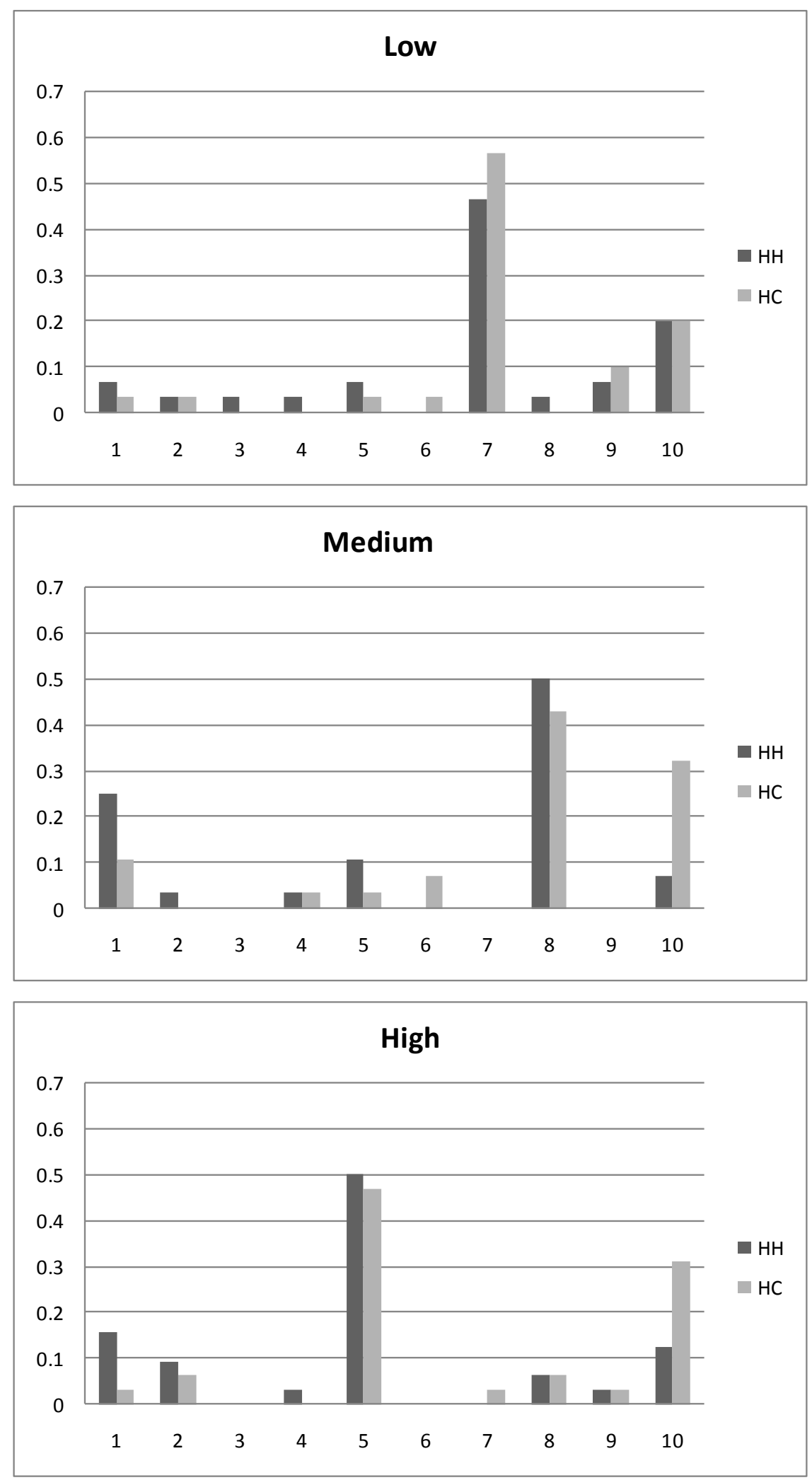

Figure 4: relative frequency distributions of strategy choices in experiment 2 


\begin{tabular}{|c|c|c|c|c|c|}
\hline Sample & Bins & $\begin{array}{r}\text { Partitione } \\
\mathrm{HH}\end{array}$ & $\begin{array}{l}\text { ibution } \\
\mathrm{HC}\end{array}$ & $\begin{array}{c}\chi^{2} \\
\text { (df) }\end{array}$ & P-value \\
\hline Experiment 1 & $\begin{array}{r}<10 \\
10\end{array}$ & $\begin{array}{l}16 \\
28\end{array}$ & $\begin{array}{r}38 \\
6\end{array}$ & $\begin{array}{c}23.2 \\
(1)\end{array}$ & $<0.01$ \\
\hline Experiment 2: Low & $\begin{array}{r}<7 \\
7 \\
>7\end{array}$ & $\begin{array}{r}7 \\
14 \\
9\end{array}$ & $\begin{array}{r}4 \\
17 \\
9\end{array}$ & $\begin{array}{l}1.1 \\
(2)\end{array}$ & 0.57 \\
\hline Experiment 2: Medium & $\begin{array}{r}<8 \\
8 \\
>8 \\
\end{array}$ & $\begin{array}{r}12 \\
14 \\
2 \\
\end{array}$ & $\begin{array}{r}7 \\
12 \\
9 \\
\end{array}$ & $\begin{array}{l}5.9 \\
(2)\end{array}$ & 0.05 \\
\hline Experiment 2: High & $\begin{array}{r}<5 \\
5 \\
>5\end{array}$ & $\begin{array}{r}9 \\
16 \\
7\end{array}$ & $\begin{array}{r}3 \\
15 \\
14\end{array}$ & $\begin{array}{l}5.4 \\
(2)\end{array}$ & 0.07 \\
\hline Experiment 2: Combined & $\begin{array}{r}<5 \\
5 \\
>5\end{array}$ & $\begin{array}{l}23 \\
21 \\
46\end{array}$ & $\begin{array}{r}9 \\
17 \\
64\end{array}$ & $\begin{array}{l}9.5 \\
(2)\end{array}$ & $<0.01$ \\
\hline
\end{tabular}

Table 3: partitioned distributions of choices in experiments 1 and 2, with Chi-squared tests

Note: In each case, $\mathrm{HH}$ and $\mathrm{HC}$ choices were combined to determine the overall mode of the distribution. The bins were then set as integers below, equal to and above this value in $\mathrm{HH}$ and $\mathrm{HC}$ separately. The requirement of the $\chi^{2}$ test that expected cell frequencies are at least 5 precludes general use of a finer partition.

Result 5. There is weak support for the bounded rationality hypothesis.

Next consider the bounded rationality hypothesis that $\mathrm{CHT}_{r}$ 's performance will improve with the complexity of the task relative to that of team reasoning theory. Prediction 5 a can be assessed using a 2-tailed $Z$ test of equality of proportions for i) Low vs Medium, ii) Low vs High and iii) Medium vs $\mathrm{High}$, in $\mathrm{HH}$, with Bonferroni correction for multiple comparison. For the team reasoning point predictions (3a), tests $\mathrm{i})$-iii) are not significant $\left(\mathrm{Z}=0.25,0.26\right.$ and 0 respectively). For the $\mathrm{CHT}_{\mathrm{r}}$ point predictions (4a), test $\mathrm{iii})$ is significant at the $5 \%$ level $(Z=2.54 ; p=0.02)$, ii) at the $10 \%$ level $(Z=2.05$; $p=0.08)$, and i) is not significant. However, this analysis is dependent on not viewing choices of $\{1\}$ in Low and Medium as attempts at best-responding to randomisation. If instead we view choices of integers in the range $[1,3]$ as cohering with $\mathrm{CHT}_{r}$ in each game, as seems natural, there is no significant difference at the $10 \%$ level for any case $(Z=1.44,1.18$ and 0.31 respectively). For prediction $5 b$, we have already noted that treatment differences emerge in Medium and High. In addition, we judge whether switches to higher and lower integers are equi -probable for each game 
separately, using a 2-tailed binomial test. In Low, 63\% of switches were to lower integers in $\mathrm{HH}$ ( $\mathrm{p}=$ 0.29), in Medium 75\% ( $p=0.02)$ and in High $82 \%(p=0.01)$. 5b is therefore supported.

In summary, point predictions favour team reasoning (3a) but there is some support for $\mathrm{CHT}_{r}$ in the sense that subjects tend to switch towards lower numbers in $\mathrm{HH}$. There is only weak support for the bounded rationality hypothesis. Conformity with either theory's point predictions does not change significantly across the 3 games, but players seem more likely to choose a higher number in $\mathrm{HC}$ than $\mathrm{HH}$ as difficulty increases.

\subsection{Interpretation of Experiment 2}

Overall the results of experiment 2 favour team reasoning over $\mathrm{CHT}_{r}$ in all three games, despite our attempt to make things difficult for team reasoning. We had expected that many subjects who choose the team reasoning strategy in $\mathrm{HC}$ would have played a low number in $\mathrm{HH}$ in the harder tasks. But only $7 \%$ of subjects behave in this manner. This apparent robustness of team reasoning suggests that coordination is not driven by beliefs about the rationality structure of the population, as posited by the $\mathrm{CHT}_{\mathrm{r}}$ account.

In spite of this, there is evidence of a slight tendency towards $\mathrm{CHT}_{\mathrm{r}}$ type behaviour as difficulty increases. It is clear from the failure of prediction $3 b$ that team reasoning cannot be the only non-random process at work generating the observed data. The support for predictions $4 \mathrm{~b}$ and $5 b$ is consistent with the strategic anticipation of unpredictable behaviour in the manner envisaged by the $\mathrm{CHT}_{r}$ account, but this is a relatively minor feature of the data. Of subjects who chose the team reasoning strategy in $\mathrm{HC}, 83 \%$ also chose it in $\mathrm{HH}$. It therefore seems unlikely that more cognitively able subjects' expectations about others' maths ability played much of a role.

A caveat to our results is that the manipulation of cognitive difficulty, as proxied by the proportion of correct choices in HC, may not have been as effective as intended. Though we lack direct measures of difficulty, the observed proportion playing the team reasoning prediction in $\mathrm{HC}$ does not monotonically decrease across Low, Medium and High. In fact these differences in proportions lack statistical significance, but compliance with team reasoning in $\mathrm{HH}$ in Experiment 1 seems higher than that in $\mathrm{HC}$ in Experiment 2 (Medium and High) with marginal significance (2-tailed Z test, $\mathrm{p}=0.09$ ). 


\section{Discussion}

The main result of this study, which is consistent across both experiments, is that the team reasoning predictions fare much better than the predictions of response to uniform randomisation in risky coordination games. It therefore seems implausible that $\mathrm{CHT}_{r}$ could account for the evidence that has been claimed for team reasoning, outlined in section 2 . When we simulate randomising players, we find differences in modal choices between HH and HC. When we simulate team reasoning, we do not. In the absence of a convincing alternative explanation of our data, the study is broadly supportive of team reasoning, though there are some features of the data it cannot explain. ${ }^{8}$

It is natural to speculate on the conditions giving rise to team reasoning, assuming this does underlie much of the data. A relevant hypothesis with empirical support is that common fate generates group identity (Campbell 1958, Brewer and Miller 1996, p42-43). In the games studied here, there is no difference in outcome between paired participants in any cell of the ga me matrix, so common fate is fully implemented. This may trigger group identity between participants even when the group is not pre-formed. Group identification might even be a default social attitude in situations where interests coincide. It seems natural to posit that group identity triggers team reasoning.

We conjectured that best-responding to randomisation was a plausible behavioural strategy where a cognitive hierarchy is likely to exist, and that this is more probable when tasks are more demanding. Therefore experiment 2 sought to increase the cognitive difficulty of the games. There is support in the data for a relatively weak tendency towards $\mathrm{CHT}_{r}$, when the tasks became more difficult. However, responding to randomisation did not become a very pronounced feature of the data. Moreover, we did not detect the difference between $\mathrm{HH}$ and $\mathrm{HC}$ treatments predicted by $\mathrm{CHT}_{\mathrm{r}}$. This suggests that $\mathrm{CHT}_{r}$ may have little behavioural significance for risky coordination games.

A further reason that the $\mathrm{CHT}_{\mathrm{r}}$ account performed relatively badly may be that uniform randomisation is not a good representation of what people do when a particular decision problem is beyond their ability to solve. This is suggested in particular by the pattern of HC choices in experiment 2, shown in Figure 4, in which the incorrect choices occurred with greater frequency above than below the correct answer. There, participants knew that their partner's strategy would be computationally correct, regardless of its difficulty. It may therefore become defensible to choose a high number, if a subject knows the solution is not a low number. For example, if a subject in High

\footnotetext{
${ }^{8}$ Capra et al (1999) and Goeree and Holt (2005) show that a stochastic generalization of an equilibrium, the Quantal Response equilibrium (QRE; McKelvey and Palfrey, 1995), characterises more choices in minimum effort and traveller's dilemma games than Nash equilibrium or potential function maxima. In Appendix 1 we compute the QRE for our risky coordination games. Given the empirically estimated "error" parameter $\mu=10$ just one QRE exists in all games we study, in which players are most likely to choose numbers $\{1\}\{2\}$ or $\{3\}$. For much lower error values, $\mu \leq 4.2$, we obtain multiple QRE; in one of these QRE players choose the team reasoning number with a very high probability.
} 
believed the answer to be 8,9 or 10 , with equal probability, they would expect equi-probable payoffs of $8,-9$, or -10 from choosing $\{8\},-9,9$ or -10 from $\{9\}$, and $-10,-10$ or 10 from $\{10\}$. Choices in the interval $[1,7]$ would be seen as dominated, $\{8\}$ as stochastically dominated, and a risk neutral subject would choose between $\{9\}$ and $\{10\}$.

It therefore seems that actual behaviour in games when people are cognitively challenged is a complex matter. For example in High, people who were not able to spot the solution may nonetheless have known that it was a number greater than, say, 3 , if they understood the mathematical notation. In $\mathrm{HH}$, they then also have to weigh the probability that their partner regards the problem as easy. This aspect of their decision is not currently represented in any version of $\mathrm{CHT}$, since $\mathrm{CHT}$ agents do not consider that others may be more sophisticated than themselves.

\section{Conclusions}

Previous studies implemented classic coordination games finding that choices cluster at PDE strategies, either of the original or transformed games. But these games elide the PDE with the best response to randomisation, so team reasoning and $\mathrm{CHT}_{r}$ make the same predictions. We therefore tested the team reasoning explanation of coordination against $\mathrm{CHT}_{r}$, using a novel experimental game designed to clearly distinguish the two accounts. In this 'risky coordination game' subjects still have to match their decisions to win prizes, but suffer variable losses if they fail. The experiments reported are also the first attempt to test team reasoning against $\mathrm{CHT}_{r}$, since previous empirical studies of $\mathrm{CHT}$ in the context of coordination assumed $\mathrm{CHT}_{\mathrm{p}}$.

Our data are consistent with team reasoning but not $\mathrm{CHT}_{\mathrm{r}}$ as the main driver of coordination. Although we studied conditions favourable to the latter by increasing the cognitive difficulty of the coordination problem $\mathrm{CHT}_{\mathrm{r}}$ still had only very limited success. It therefore seems that the explanatory mechanisms for focal points compatible with the evidence across all relevant experiments are i) team reasoning and ii) psychological salience. Our results count against $\mathrm{CHT}_{\mathrm{r}}$ and the principle of insufficient reason as explanations of play in classic coordination problems.

Team reasoning therefore remains a key ingredient of the explanation of coordination game data. To that extent, a question mark is raised again st methodological individualism, in the sense of a commitment to explaining actions with reference only to reasons addressed to individuals. If a propensity to team reason became firmly empirically established, this would have wider implications. Team reasoning has also been invoked to explain play in prisoners' dilemma and public good games (Bacharach, 2006), which serve as models of a great many important real-world situations. We therefore also judge that team reasoning should receive more attention from 
economic researchers. On the other hand, it is possible that further individualistic explanations of coordination game data will be proposed and will merit testing.

One fruitful avenue for further team reasoning research might be to go beyond choice data. In common with the great majority of behavioural economics experiments, our trials test predictions of choices but do not provide direct evidence about cognitive processes. There is therefore also a role for qualitative or possibly neurological research in future, to probe the team reasoning hypothesis more directly.

\section{References}

Agresti, A. (1996). An Introduction to Categorical Data Analysis. Wiley: Chichester.

Bacharach, M. (2006). N. Gold and R. Sugden (Eds.) Beyond Individual Choice. Teams and Frames in Game Theory. Princeton University Press: Princeton, N.J.

Bacharach, M. (1999). Interactive team reasoning, a contribution to the theory of cooperation Research in Economics, 53, 117-147

Bacharach, M. and Bernasconi, M. (1997). The variable frame theo ry of focal points: an experimental study. Games and Economic Behavior, 19, 1-45

Bacharach, M. and Stahl, D.O. (2000). Variable-frame level-n theory. Games and Economic Behavior, $33,220-46$.

Bardsley, N., Mehta, J., Starmer, C., Sugden, R., 2010. Explaining Focal Points: Cognitive Hierarchy Theory versus Team Reasoning. The Economic Journal, 120, 40-79.

Bergstrom, T., Blume, L. and Varian, H. (1986). On the private provision of public goods. Journal of Public Economics.

Bjerring, A. (1978). The tracing procedure and a theory of rational interaction. In C.A. Hooker, J. Leach, \& E. McClennen (Eds.), Foundations and Applications of Decision Theory. Reidel: Dordrecht.

Blume, A. and Gneezy, U. (2010). Cognitive forward induction and coordination without common knowledge: An experimental study. Games and Economic Behavior, 68, 488-511

Brewer, M.B. and Miller, N. (1996). Intergroup Relations. Oxford University Press: Oxford.

Camerer, C.F., Ho, T. and Chong, K. (2004). A cognitive hierarchy model of games. Quarterly Journal of Economics, 119, 861-898.

Campbell, D.T. (1958). Common fate, similarity, and other indices of aggregates of persons as social entitites. Behavioral Science, 3, 14-25

Capra, M., Goeree, J.K., Gomez, R. and Holt C. (1999). Anomalous Behavior in a Travelers Dilemma?. American Economic Review, 89(3), 678-690. 
Crawford, V.P., Gneezy, U. and Rottenstreich, Y. (2008). The power of focal points is limited. Even a degree of payoff asymmetry may yield large coordination failures. American Economic Review, 98, 1143-1158.

Elster, J. (1982). The case for methodological individualism. Theory and Society, 11, 453-482.

Elster, J. (1985). Making Sense of Marx. Cambridge University Press: Cambridge.

Gintis, H. (2003). A critique of team and Stackleberg reasoning. Behavioral and Brain Sciences, 26, $160-161$.

Goeree, J.K. and Holt, C.A. (2005). An Experimental Study of Costly Coordination. Games and Economic Behavior, 51(2), 349-364.

Harsanyi, J. and Selten, R. (1988). A General Theory of Equilibrium Selection in Games. MIT Press: Cambridge, MA.

Hollis, M. (1998). Trust within Reason. Cambridge University Press: Cambridge

Kahneman, D. and Tversky, E. (1979). Prospect theory: an analysis of decision under risk. Econometrica, 47, 263-292.

Lewis, D. (1969). Convention: a Philosophical Study. Harvard University Press: Cambridge, MA.

McKelvey, R.D. and Palfrey, T.R. (1995). Quantal Response Equilibria for Normal Form Games. Games and Economic Behavior, 10, 6-38.

Mehta, J. Starmer, C. and Sugden, R. (1994). The nature of salience: an experimental investigation. American Economic Review, 84, 658-673.

Monderer, D. and Shapley, L.S. (1996). Potential games. Games and Economic Behavior, 14, 124143.

Nagel, R. (1995). Unravelling in guessing games: an experimental study. American Economic Review, 85, 1313-1326.

Pawson, R. and Tilley, N. (1997). Realistic Evaluation, Sage: London.

Regan, D.H. (1980). Utilitarianism and Cooperation. Clarendon Press: Oxford.

Schelling, T. (1960). The Strategy of Conflict. Harvard University Press: Cambridge, MA.

Skyrms, B. (1989). Correlated equilibria and the dynamics of rational deliberation. Erkenntnis, 31, 347-364.

Stahl, D.O. (1993). Evolution of smart ${ }_{n}$ players. Games and Economic Behavior, 5, 604-617.

Stahl, D.O. and Wilson, P. (1995). On players' models of other players. Games and Economic Behavior, 10, 218-254.

Sugden, R. (1984). Reciprocity: the supply of public goods through voluntary contributions. The Economic Journal, 94, 772-787.

Sugden, R. (1993). Thinking as a team: towards an explanation of nonselfish behaviour. Social 
Philosophy and Policy, 10, 69-89.

Sugden, R. (1995). A theory of focal points. The Economic Journal, 105, 533-550. 


\section{Appendix 1}

\section{Proof of $\mathrm{CHT}$ Predictions for Experiment 1}

Let $j$ denote the opponent's chosen integer. The difference in utility, defined over experimental tokens, from choosing $\{i+1\}$ over $\{i\}$ is:

0 if $j>i+1$

$\mathrm{U}(i+1)-\mathrm{U}(-\mathrm{i}-1)$ if $j=i+1$

$\mathrm{U}(-\mathrm{i}-1)-\mathrm{U}(\mathrm{i})$ if $j=i$

$\mathrm{U}(-i-1)-\mathrm{U}(-\mathrm{i})$ if $j<i$

If $U^{\prime}(i)>0$ for all $i$ and the player evaluates equally the probabilities that its opponent chooses any strategy $\{j\}$ then we can ignore probabilities and probability weights. It follows that

$$
\{i+1\} \stackrel{\succ}{\sim}\{i\} \leftrightarrow U(i+1)-U(i)+(i-1)[U(-i-1)-U(-i)] \stackrel{>}{=}=0
$$

For $i=1$ this reduces to $U(2)-U(1)>0$, thus strategy $\{2\}$ is always preferred to strategy $\{1\}$. Strategy $\{1\}$ is in fact stochastically dominated by strategy $\{2\}$. For strategies $\{2\}, \ldots,\{10\},(1)$ implies $\{i+1\}$ is weakly preferred to $\{i\}$ if and only if

$U(i+1)-U(i) \geq(i-1)[U(-i)-U(-i-1)]$

Consider $i \geq 3$. Under EUT with either risk aversion or risk neutrality, and also under Prospect Theory, $U(i+1)-U(i) \leq U(-i)-U(-i-1)$. Therefore (2) is not satisfied, and strategy $\{i\}$ is preferred to strategy $\{i+1\}$. Hence, under standard models of choice under risk, strategies $\{2\}$ and $\{3\}$ are preferred to all other strategies.

Next, consider $i=2$. Under risk neutrality (2) holds with equality because of the assumption that $U^{\prime}=k$, so $\{2\} \sim\{3\}$. Under risk aversion $U^{\prime \prime}<0$, and under Prospect Theory $U^{\prime}(x)<U^{\prime}(-x)$. Either assumption implies that (2) does not hold, so $\{2\}$ is strictly preferred to $\{3\}$.

Finally, as under risk neutral EUT, if each player believes that the other applies the principle of insufficient reason, then from an interim conclusion that $\{2\} \sim\{3\}$, it follows that $\{3\}$ is preferred, since $\{3\}$ is the best response to a $50 / 50$ chance that $j=\{2\}$ and $j=\{3\}$. Under $\mathrm{CHT}$, if for level 1 players $\{2\} \sim\{3\}$ then for levels 2 and above $\{3\}$ is preferred, if agents at those levels infer equi-probable choices from indifference at lower levels. The distribution should therefore have a single mode at $\{3\}$, with the relative frequencies of $\{2\}$ and $\{3\}$ depending on those of level 1 and higher-level players. Parallel derivations can be given of $\mathrm{CHT}$ predictions in experiment 2. 


\section{Quantal Response Equilibria for risky coordination games.}

In the discrete choice QRE players pick an action with the probability that corresponds to the exponential of its expected payoff. Let $\pi=A p$ be the vector of expected payoffs in the game given by matrix $A$, when $p$ is the vector describing probabilities that the opponent will pick different actions in the game. The player will choose an action according to the 'logit response' (McKelvey and Palfrey, 1995), with the probability proportional to the exponential of its expected payoff, weighted by the error parameter $\mu: d_{i}=\exp \left(\pi_{i} / \mu\right)$. When $\mu \rightarrow 0$ the model describes rational choice, while as $\mu \rightarrow \infty$ the behaviour converges to uniform randomization. In the symmetric QRE the player and the opponent use the same probabilities, that is, $p_{i}=d_{i} / \sum_{k=1}^{n} d_{k}$.

We follow Capra et al. (1999) and find QRE in our four risky coordination games by simulating logit response dynamics. The code used for the simulation in Mathematica is available on request from the authors. Starting from several thousand randomly drawn initial probability distributions over the possible game actions, we repeatedly calculate the logit response until we detect that the dynamics has reached a fixed point where the distribution stops changing. This yields the set of QRE and their basins of attraction. For each game we find the QRE for the error parameter $\mu=10$, suggested by Capra et al. (1999). For this parameter we find that all our games have a unique QRE in which players choose any number in $\{1,2,3\}$ with a higher probability than any higher number.

Other QRE which put a high probability on the team reasoning number exist only for much lower error parameters $\mu$. For each game we estimate the maximal value of $\mu$ that permits a QRE in which the team reasoning number is played with the highest probability, by repeatedly decreasing $\mu$ in seps of 0.1 anf repeating the above procedure. For our four risky coordination games we estimate these maximal errorvalues and corresponding QRE probabilities to pick the team reasoning number as:

- baseline: $\quad \mu=4.2, \quad p_{10}=0.87$

- low: $\quad \mu=3, \quad p_{7}=0.87$

- medium: $\quad \mu=3.4, p_{8}=0.86$

- high: $\quad \mu=2.3, \quad p_{5}=0.87$

In each of the above cases there exists a second QRE where players choose any number in $\{1,2,3\}$ with a higher probability than any higher number. This QRE has by far the largest basin of attraction. The QRE analysis above suggests that the likelihood to observe team reasoning decreases as the games get harder and the players' actions and beliefs become more noisy. 